\title{
Systemic thromboembolism from a misdiagnosed non-bacterial thrombotic endocarditis in a patient with lung cancer: A case report
}

\author{
FABIANA PERRONE ${ }^{1}$, ANDREA BIAGI ${ }^{2}$, FRANCESCO FACCHINETTI ${ }^{1,3}$, \\ FRANCESCA BOZZETTI $^{4}$, ANDREA RAMELLI ${ }^{5}$, ANTONELLA VEZZANI ${ }^{5}$, TULLIO MANCA ${ }^{5}$, \\ LETIZIA GNETTI $^{6}$, MARIA MAJORI $^{7}$, VERONICA ALFIERI $^{8}$ and MARCELLO TISEO ${ }^{1,9}$ \\ ${ }^{1}$ Medical Oncology Unit and ${ }^{2}$ Cardiology Department, University Hospital of Parma, \\ I-43126 Parma, Italy; ${ }^{3}$ National Institute of Health and Medical Research, Gustave Roussy Cancer Campus, \\ University of Paris-Saclay, 94800 Villejuif, France; ${ }^{4}$ Department of Neuroradiology; \\ ${ }^{5}$ Cardiac Surgery Intensive Care Unit; Units of ${ }^{6}$ Pathology, ${ }^{7}$ Pulmonology and Thoracic Endoscopy, \\ and ${ }^{8}$ Respiratory Disease and Lung Function; ${ }^{9}$ Department of Medicine and Surgery, \\ University of Parma, I-43126 Parma, Italy
}

Received August 9, 2019; Accepted March 5, 2020

DOI: $10.3892 / 01.2020 .12056$

\begin{abstract}
Thromboembolic events are frequent in patients with cancer, commonly involving the venous and pulmonary circulation. The arterial system is rarely implicated in embolism and, when involved, a cardiogenic origin should always be excluded. In the present study, a case of a patient who developed multiple embolic events concomitantly with the diagnosis of locally-advanced non-small cell lung cancer with high expression levels of programmed death-ligand 1 (PD-L1) in $>50 \%$ of tumor cells is reported. A cardiac defect interpreted as a patent foramen ovale required low molecular weight heparin administration. Despite the anti-coagulant therapy, before first-line anticancer treatment with pembrolizumab immunotherapy could be administered due to high PD-L1 expression levels, a new hospitalization was required due to the onset of novel ischemic manifestation. New transthoracic and transesophageal echocardiography revealed a previously misdiagnosed vegetation of the mitral valve that caused systemic embolization. The lack of any sign of infection led to the diagnosis of a non-bacterial thrombotic endocarditis (NBTE), whose embolic sprouting gave rise to the widespread ischemic events. No active anticancer treatment was feasible
\end{abstract}

Correspondence to: Dr Francesco Facchinetti, National Institute of Health and Medical Research, Gustave Roussy Cancer Campus, University of Paris-Saclay, 114 Rue Edouard Vaillant, 94800 Villejuif, France

E-mail: Francesco.FACCHINETTI@gustaveroussy.fr

Key words: immunotherapy, marantic endocarditis, non-bacterial thrombotic endocarditis, non-small cell lung cancer, pembrolizumab, systemic embolism due to the rapid progression of the disease. NBTE can evolve quickly, eventually preventing any chance of treatment targeting the primary cause, which in the present study was lung cancer. If NBTE can be correctly diagnosed sooner then there may be the potential for anticancer therapy that does not worsen the hypercoagulability state, thus improving cancer-associated survival.

\section{Introduction}

The hypercoagulability state is a common condition in patients with cancer, contributing to tumor-related morbidity and mortality (1), or even revealing silent malignancies (2). A close association between cancer and thrombophilic states exists, both physio-pathologically and clinically $(1,3-5)$. Indeed, it is known that an imbalance between coagulation and fibrinolytic system happens in malignancy, because the cancer cells are able to produce and release procoagulant factors, such as tissue factors, cancer procoagulants, fibrinolityc protein (urokinase and tissue plasminogen activator), citokines (including tumor necrosis factor- $\alpha$ and interleurk-1 $\beta$ ) and antiangiogenetic molecules (1,3-5). Moreover, cancer cells interact with host vascular and blood cells, including endothelial cells, leukocytes and platelets, and these mechanisms lead to signalling cascades resulting in coagulation activation and thrombi formation (1).

Thromboembolic events can affect the venous or/and the arterious circulation (3). Venous thromboembolism is found in 10-20\% of patients with cancer and the incidence is 4-7 times higher compared with patients without cancer. Deep-vein-thrombosis (DVT) is the most common manifestation (6). The incidence of arterial thromboembolism ranges from 1 to $4.7 \%$ and non-bacterial thrombotic endocarditis (NBTE) is a rare manifestation (7). The incidence of NBTE is unknown and often is a post-mortem finding, occurring in $1.6 \%$ in the 
adult autopsies (6). Several factors are associated with increased risk of arterial and venous thromboembolic events, including cancer-associated factors, patients-associated factors and treatment-associated factors (3,7). Among cancer-associated factors, lung, gastric and pancreatic cancer, especially in advanced stages, are associated with a higher risk of thromboembolic events $(3,8-10)$. Patient-associated factors contributing to higher risk include older and female patients with low performance status and history of viral or bacterial infection, chronic kidney disease, pulmonary disease and obesity $(3,7)$. Meanwhile, treatment factors associated with increased risk of thrombi formation include chemotherapy and antiangiogenetic drugs, hormonal therapy, prolonged hospitalization or central venous catheters (7).

The treatment depends on the severity of thrombotic event; a massive pulmonary embolism could be fatal and requires early hospitalization, whereas mild to moderate cases are treated with anticoagulant therapy, namely unfranctioned heparins (fondaparinux) or low molecular weight heparin (7). Recently, the Hokusai VTE Cancer Trial demonstrated that non-inferiority of Edoxaban, an oral factor Xa inhibitor, for at least 6 months and up to 12 months compared to subcutaneous deltaparin in cancer patients with recurrent venous thromboembolism (11). Thus, Edoxaban could be a valid alternative to unfranctioned heparins (fondaparinux) or low molecular weight heparin.

The present study reports a case of a patient presenting with a newly diagnosed locally advanced non-small cell lung cancer (NSCLC) who developed widespread thromboembolisms that prevented any chance of local or systemic therapy. An underlying NBTE, previously misdiagnosed as a patent foramen ovale (PFO), was deemed responsible for the systemic hypercoagulability state.

\section{Case report}

Onset of embolic events. At December 12, 2018, a 63-year-old male with a history of smoking (20 packs/year) and lacking significant comorbidities developed DVT in the left leg and started rivaroxaban anticoagulant therapy at a dose of $10 \mathrm{mg} /$ daily. On 24th December 2018, the patient was hospitalized in an Italian communitry hospital due to the abrupt onset of dyspnea. A thoracic CT scan confirmed pumlonary thromboembolism (PTE), documented as a mass in the lower lobe of the right lung, with ipsilateral ilo-mediastinal and infra-clavear pathological lymph nodes (Fig. 1A and B), evocative of lung cancer with lymph nodal spreading. In addition, an abdominal CT scan demonstrated signs of splenic and renal infarctions (Fig. 1C and D).

A total of 5 days later, the patient developed right cerebellar signs associated with confusion, including nystagmus, ataxia, dysmetria and adiadochokinesia. An MRI of the brain revealed multiple diffuse bi-hemispheric cerebral and cerebellar areas, both in the grey matter (especially of the occipital lobes) and in the deep cerebral white matter, hyperintense in fluid-attenuated inversion recovery/fast spin echo sequences when using enhanced contrast (Fig. 2A-I). These lesions were characterized by diffusion restriction and demonstrated moderate patchy and gyriform enhancement. Given the recent medical history of the patient, differential diagnoses were hypothesized, including ischemic lesions on an embolic basis and miliary brain metastases, although this was less likely the radiology picture. Transthoracic echocardiogram (TTE) did not show any valvular lesion or other anatomical defects. Because of the critical patient condition, a transesophageal echocardiogram (TEE) was not performed. In the following days, the patient experienced spontaneous rapid improvement of the neurological state with complete resolution of confusion and cerebellar symptoms.

In view of the neuro-radiological imaging and the positive evolution of neurological condition, a cardio embolic origin of the brain lesions was suspected. A TEE was carried out and revealed a PFO that was assumed to be the cause of the paradoxical arterial embolisms. No surgical correction of PFO was envisaged by heart-surgeon, so, at January 10, 2019, the patient was discharged and prescribed subcutaneous low-molecular-weight heparin (LMWH) at dose of 6,000 IU twice daily.

Lung cancer diagnosis and staging. Cancer staging was completed using a PET scan, which confirmed the locally advanced extension of the disease (IIIB stage according to Tumor-Node-Metastasis staging system) (12) without distant metastases. An endobronchial ultrasound (EBUS)-guided transbronchial needle aspiration was performed, allowing the diagnosis of a large-cell carcinoma of the lung (hematoxylin and eosin staining). Immunohistochemistry (IHC) did not differentiate between squamous or non-squamous type, was positive for cytokeratin pool and lacked expression of thyroid transcription factor-1 (TTF-1), p40, chromogranin A and synaptophysin (Fig. 3A-C) (13). The positivity for cytokeratin CAM5.2 (data not shown) excluded the possibility of a lymphoma. Neither EGFR mutations, researched with Therascreen RGQ RT-PCR kit (Qiagen, Inc; qPCR) nor $A L K$ gene rearrangements (IHC, D5F3 clone) were detected. All the procedures for the molecular analysis have been performed following the specific manufacturer's instructions on the tumor material obtained by the EBUS-guided needle aspiration (14). IHC demonstrated high positivity of programmed death-ligand 1 (PD-L1), as PD-L1 tumor proportion score was 55\% (Fig. 3D). The patient was therefore a candidate for immunotherapy with pembrolizumab, an anti-programmed death-1 antibody (15).

Novel ischemic events leading to NBTE detection. Only a few days before initiation of pembrolizumab treatment, the onset of diplopia and gait impairment required a new hospitalization (University Hospital of Parma, Italy). With the limitation of a comparison between different imaging techniques, the first CT scan already documented evolution of the lesion when compared with the MRI performed 15 days before during the first hospitalization (Fig. 4 vs. Fig. 2).

One day later, the patient developed substernal chest pain and dyspnea; however, body temperature, blood pressure and heart rate were regular. Cardiac auscultation revealed a diastolic murmur consistent with aortic regurgitation, without any other notable symptoms at the physical examination. A 12-lead electrocardiogram showed sinus tachycardia (90 beats/min) with ST segment elevation in DII-DIII-aVF derivations. Laboratory tests revealed elevated serum troponin I with a peak value of $22.91 \mathrm{ng} / \mathrm{ml}$ (normal value $<0.006 \mathrm{ng} / \mathrm{ml}$ ) (16). TTE showed a 

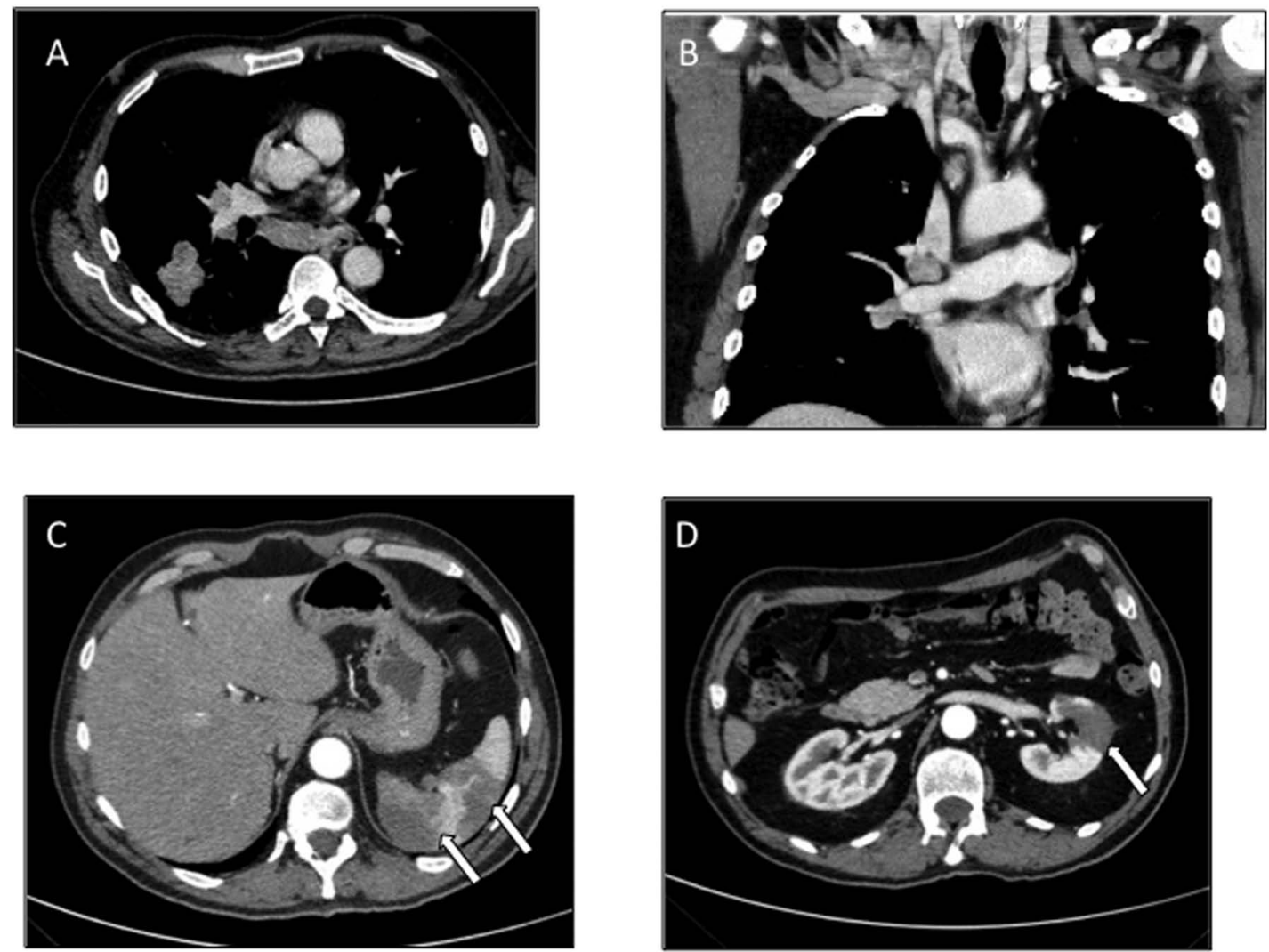

Figure 1. Baseline CT scan. (A and B) Thoracic CT scan showed a mass in the lower lobe of the right lung, with ipsilateral ilo-mediastinal (subcarinal) and right infra-clavear pathological lymph nodes. (C and D) Abdominal CT scan documented signs of previous, non-datable multiple splenic and renal infarctions (white arrows), likely attributable to an acute/subacute phase of infarction.

reduced left ventricular ejection fraction (LVEF) of $45 \%$, a mobile mass on the anterior leaflet of mitral valve and a moderate aortic insufficiency. The following development of acute pulmonary edema with respiratory failure required noninvasive ventilation. Coronary angiography showed reduced flow (Thrombolysis in Myocardial Infarction score=2) (17) of the recurrent branch of the descending artery rapidly after injection of the contrast agent, whose appearance was consistent with an embolus. No atherosclerotic lesions were observed in the remaining vessels. Due to the hemodynamic and respiratory instability, the patient was admitted to the intensive care unit. Therapy with aspirin and $\beta$-blockers was initiated while maintaining LMWH treatment. Suspicion of infective endocarditis led to blood culture tests as well as empiric intravenous antibiotic therapy with linezolid $600 \mathrm{mg}$ twice daily, daptomycin $6 \mathrm{mg} / \mathrm{kg}$ once daily and piperacillin/tazobactam $4.5 \mathrm{~g}$ three times daily. Clinical examination at this timepoint revealed petechiae on the skin of the lower limbs, which is highly suggestive of peripheral embolization (18).

An abdominal CT scan was performed at the onset of abdominal pain and loss of blood in the stool, revealing occlusion of a distal branch of the superior mesenteric artery, accompanied by the thinning of the corresponding ileal loop (Fig. 5A and B). The patient underwent emergency segmental ileo-cecal resection, with the histological diagnosis of bowel infarction (hematoxylin and eosin staining; Fig. 5C and D) and remained intubated thereafter.

A TEE was performed following clinical stabilization and revealed a mobile echogenic mass of $0.8 \times 0.6 \mathrm{~cm}$ on the atrial surface of the anterior leaflet of the mitral valve, moderate aortic insufficiency and reduced LVEF (40\%) (Fig. 6). No signs of PFO were detected.

The negativity of blood cultures and the normal values for inflammatory markers (procalcitonin $0.26 \mathrm{ng} / \mathrm{ml}$, range $0.00-0.5 \mathrm{ng} / \mathrm{ml})$,togetherwiththediffuseembolicmanifestations led to a final diagnosis of NBTE.

The patient then went into a coma, with an additional brain CT scan documenting increased number and dimension of the aforementioned described bi-hemispheric infarct lesions in different phases of evolution, compatible with the progression of the cerebral ischemic events and the onset of novel interested foci (Fig. 4C-E).

Then, the patient developed multi-organ failure and passed away, 70 days after lung cancer diagnosis. Post-mortem examination was not carried out.

\section{Discussion}

NBTE is a rare but serious manifestation of the hypercoagulability state in patients with cancer, observed in $\sim 1.25 \%$ of autoptic cases compared with $0.2 \%$ of autopsies performed in patients without cancer (16). Adenocarcinoma histology is frequently involved in the occurrence of NBTE with pancreatic adenocarcinoma being the most common $(6,19)$.

The pathogenesis of NBTE endocarditis is not completely understood. The local and systemic milieu of pro-thrombotic factors secreted by tumors, such as tumor necrosis factor, 

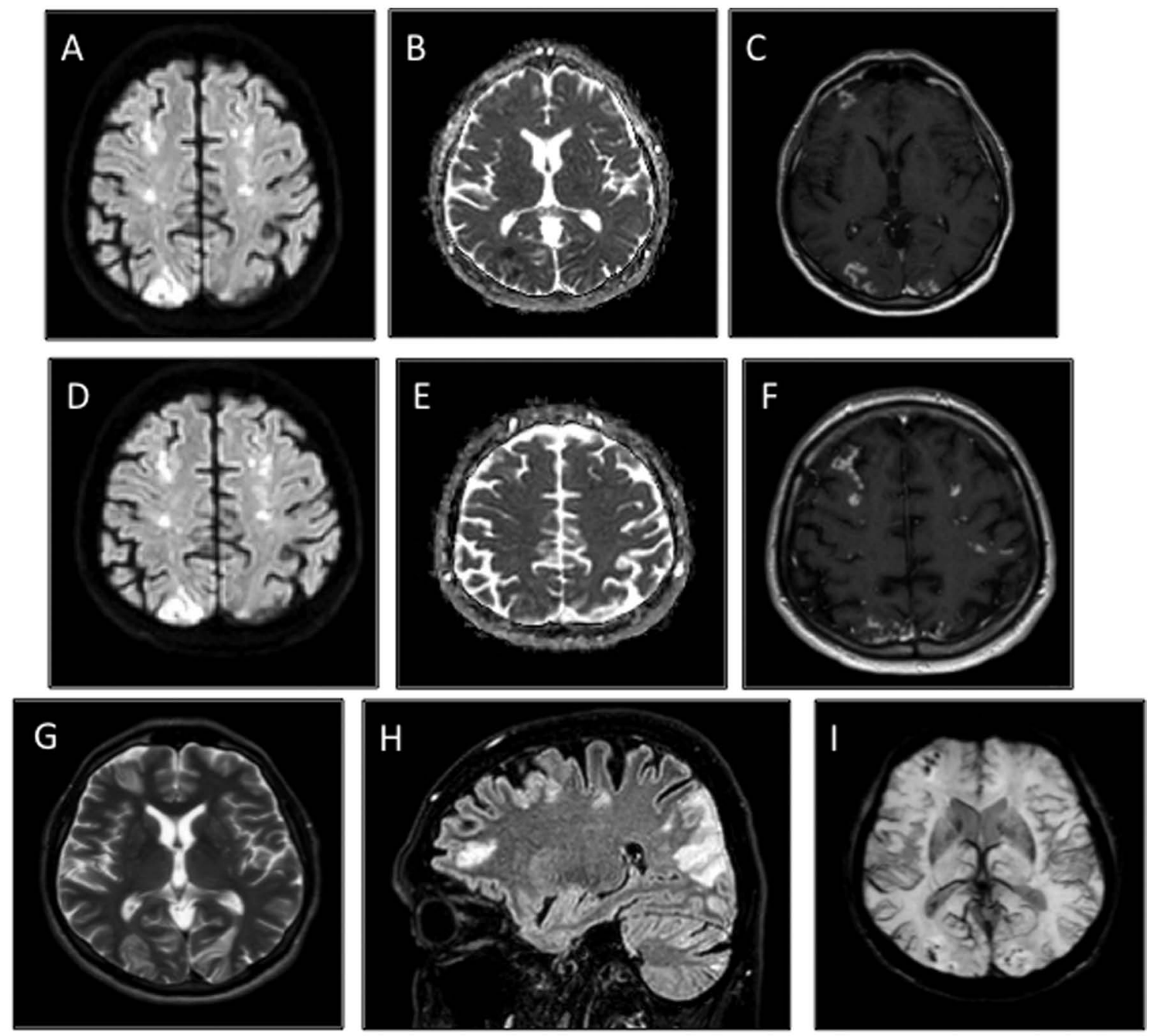

Figure 2. Baseline brain MRI. (A-C) Multiple patchy foci of diffusion restriction in the grey matter of the occipital lobes, consistent with acute cerebral infarcts. The multiple patchy foci affected not only the distal territories of the both posterior cerebral arteries but also innumerable tiny foci restricted diffusion in the deep cerebral white matter. (D-F) Star field pattern characteristic of cerebral embolism syndrome. (C and F) Moderate patchy and gyriform enhancement (sign of subacute cerebral infarcts) were evidenced after gadolinium administration. (A and D) Diffusion weighted imaging. (B and E) Apparent diffusion coefficient maps. (C and F) T1-w post gadolinium administration images. (G and H) Overview of the multiple ischemic infarcts as (G) FSE and (H) FLAIR hyperintensities in T2-w images. (I) Susceptibility weighted imaging showed several tiny blooming hypointense foci throughout the hemispheric grey and white matters, characteristic of microbleeds secondary to cerebral embolism syndrome.

interleukin 1, tissue factor and cancer procoagulant, are likely to be involved in the development and maintenance of cardiac vegetations (1). Cardiac vegetations are composed of aseptic thrombi, lacking inflammatory reaction and growing on valvular surface. Aortic and mytralic valves are the most commonly implicated, while the valves of the right side of the heart are less frequently affected (20).

In patients with cancer, embolization occurs in around 50\% of NBTE cases (21). Notably, NBTE leads to embolization events more frequently compared with bacterial forms, given their mild cellular composition and the weaker intercellular links (22). The spleen, kidneys, brain, mesenteric and coronary arterial circulation are the most commonly structures implicated in NBTE $(21,23,24)$.

The peculiarity of the present case was the development of systemic embolisms in a short period of time, while undergoing LMWH for a misdiagnosed PFO. Asymptomatic kidney and splenic infarctions were the first to be detected concomitantly with DVT, PTE and cerebral dissemination.
The venous and pulmonary circulation is less frequently involved in NBTE embolization but are the most common manifestations of the hypercoagulability state (6). Therefore, differential pathogenetic mechanisms of NBTE on the basis of DVT and PTE cannot exclude. After presenting with these thromboembolic manifestations, the patient then developed cardiac, mesenteric and lower limb ischemia. Notably, the last brain CT scan (Fig. 4) documented a variety of lesions compatible with strokes occurring at different time points, suggesting that there were continuous emboli shedding from the valvular vegetations.

TEE, the best diagnostic tool to identify bacterial or non-bacterial endocarditis, has higher sensitivity compared with TTE $(25,26)$. In the present case, TTE did not show abnormalities. Otherwise, the TEE documented a different cardiac involvement in terms of a PFO as the origin of the embolic foci. Both TTE and TEE, performed 40 days later, demonstrated sub-centimetric vegetation on the mitral valve, which explained the development of the cardiogenic thromboembolic state. 

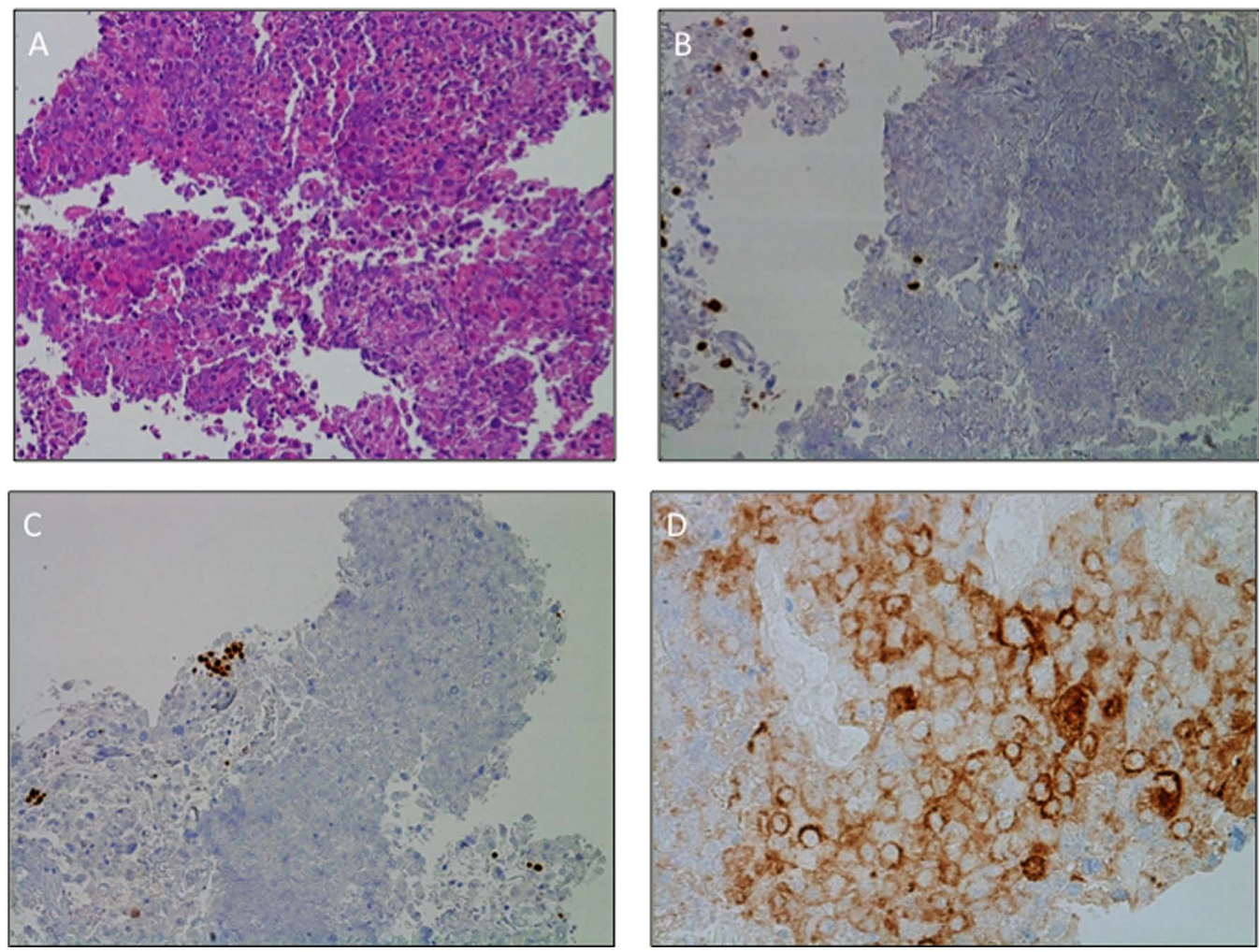

Figure 3. Morphological and immunohistochemistry diagnosis of large-cell carcinoma of the lung. (A) Hematoxylin and eosin staining documenting malignant large pleomorphic cells with a moderate to abundant amount of cytoplasm, vesicular nuclei, prominent nucleoli and necrosis. IHC staining of (B) TTF1 and (C) p40 revealed expression of the two markers on pneumocytes (TTF1) and basal cell (p40) only, thus supporting the diagnosis of large-cell carcinoma of the lung. The negativity of TTF1 and p40 precluded the diagnosis of lung adenocarcinoma or squamous cell carcinoma, respectively. Tumors lacked morphological and immunohistochemical evidence of glandular, squamous or neuroendocrine differentiation. (D) Programmed death-ligand 1 expression levels on the surface membrane of 55\% of tumor cells. Magnification, (A-C) x20 and (D) x40. TTF1, transcription termination factor 1.
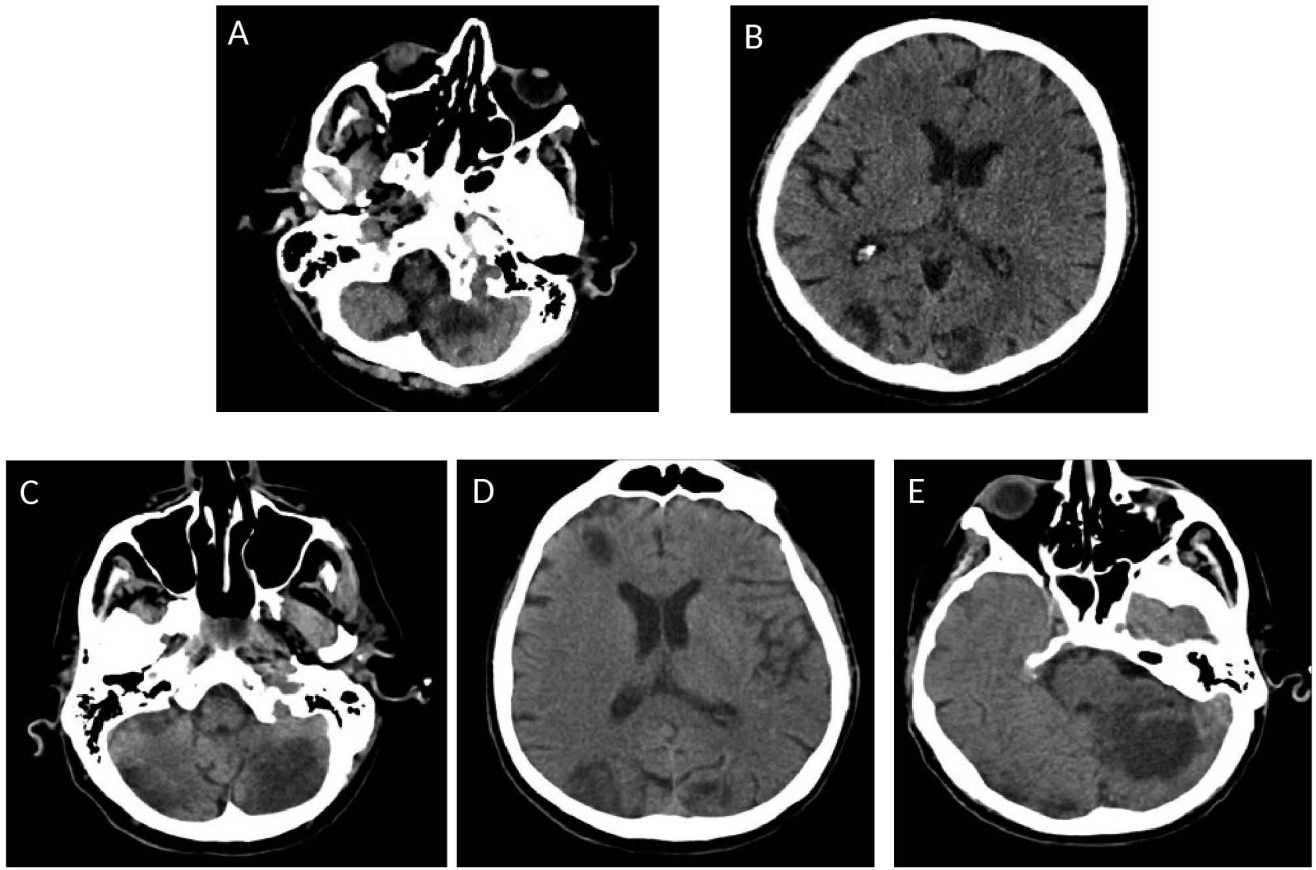

Figure 4. Comparison of brain CT scans performed with 20-days interval. (A and B) At hospitalization. (C, D and E) After 20 days. (A and C) Left antero-inferior cerebellar ischemic infarcts appeared more demarcated and bigger in the most recent exam (B), documenting a new ischemic lesion on the antero-inferior right cerebellar hemisphere in the acute phase of ischemia. (B and D) Bilateral occipital ischemic infarcts too appeared more demarcated and bigger. (E) Another new ischemic lesion in an acute phase was detected in the left postero-superior cerebellar hemisphere. 

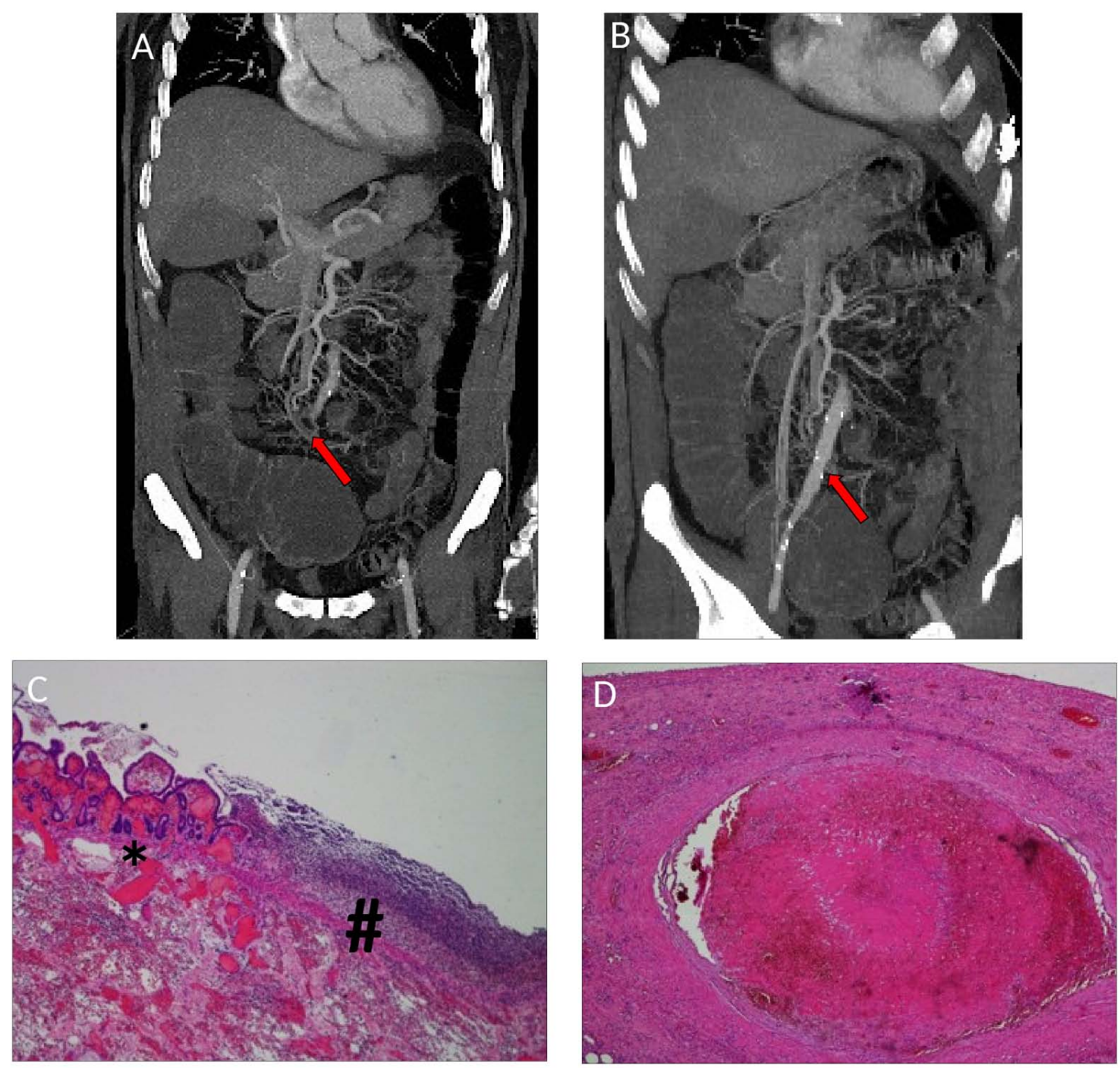

Figure 5. Radiological and pathological evidence of bowel ischemia. (A and B) Abdominal CT scan performed at the acute onset of abdominal pain and loss of blood stool documented the occlusion of a distal branch of the superior mesenteric artery, accompanied by the thinning of the corresponding ileal loop (red arrows). (C) Hematoxylin and eosin staining at magnification, $\mathrm{x} 4$ of the interface between the intestinal mucosa with hemorrhagic infarction ("on the left) and the wide superficial inflammation ( ${ }^{*} \mathrm{on}$ the right). (D) Hematoxylin and eosin staining at magnification, $\mathrm{x} 4$ of the mesenteric artery thrombosis in cause of the bowel ischemia.
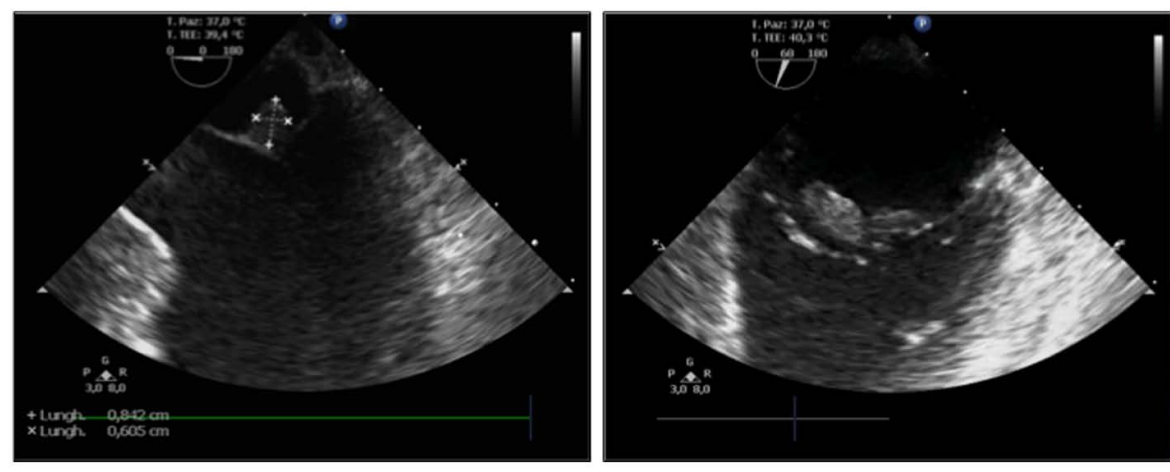

Figure 6. A transesophageal echocardiogram performed during the second hospitalization revealed a mobile echogenic mass of $0.8 \mathrm{x} 0.6 \mathrm{~cm}$ on the atrial surface of the anterior leaflet of the mitral valve, moderate aortic insufficiency and reduced left ventricular ejection fraction by $40 \%$.

Considering this, the importance of performing TEE by experienced physicians to rule out the possibility of endocarditis should be reiterated, particular when accompanying symptoms, radiological features and medical history (for example history of malignancy, as in the present case, or recurrent thrombotic events) are suggestive of NBTE. 
The treatment of NBTE relies on therapy targeting the underlying pathology, for example lung cancer in the present case, but inflammatory chronic diseases (including systemic lupus erythematosus or connective tissue disorders) can also be a cause (27). NBTE also relies on anticoagulant therapy (6). Surgical intervention is not recommended for NBTE in patients affected by advanced and non-curable cancer (28). Both unfractionated heparin and LMWH are potentially effective in decreasing the risk of embolization (6). On the other hand, vitamin $\mathrm{K}$ antagonists, such as warfarin, fail to prevent the systemic emboli in NBTE, potentially due to the not involvement of vitamin K-dependent factors (factors II, VII, IX and $\mathrm{X}$ and protein $\mathrm{C}$ and $\mathrm{S}$ ), inducing the trombophilic state in NBTE (29). There is no definite evidence demonstrating the efficacy of fondaparinux, a synthetic pentassaccharide like heparin, for the treatment of NBTE. Instead, direct oral anticoagulants, such as rivaroxaban and edoxaban, are acceptable alternatives to LMWH but should be used carefully, due to the considerable risk of bleeding events (30). However, recently a case of metastatic pancreatic cancer underlying a NBTE was reported, highlighting the clinical improvement and tumor control obtained with LMWH and chemotherapy administration (31).

Unfortunately, in the present case, LMWH administration for the suspected PFO was not sufficient to avoid diffuse embolization, even at the curative dose. Although, the patient was a candidate for Pembrolizumab therapy due to locally advanced disease stage (IIIB) and high expression levels of PD-L1 ( $\geq 50 \%$ ) (15), multi-organ failure due the multiple foci embolization did not allow sufficient time for specific anticancer therapy to be administered.

NBTE should be strongly suspected when a patient with underlying malignancy experiences multiple arterial thrombotic events. Diagnosis of NBTE should not be excluded if the TTE does not demonstrate cardiac anomalies and a TEE should still be performed, given the risk of false negatives (32). In hindsight, the precocious putative detection of a NBTE in the present patient could have sped up the diagnosis and improved patient care, for example prolonging hospitalization with close clinical monitoring. Moreover, the current availability of targeted and immunotherapy agents, such as tyrosine kinase inhibitors and pembrolizumab, respectively, are characterized by lacking significant pro-thrombotic effects compared with traditional cytotoxic agents. So, these drugs in the treatment of patients with NBTE and cancer are deemed safe (33).

On the other hand, due to the strong association between cancer and NBTE, an unknown neoplastic disease should be considered for patients who develop systemic embolisms from sterile cardiac vegetations. It should be mandatory to suspect NBTE in these cases. NBTE quickly worsens and can be fatal if left untreated and the early start of adequate anticoagulant and specific anticancer therapy may improve survival and risk of complications due to NBTE.

\section{Acknowledgements}

The authors would like to thank Dr Tala Tayoun (National Institute of Health and Medical Research, University Paris-Saclay) for her medical writing assistance.

\section{Funding}

No funding was received.

\section{Availability of data and materials}

All data generated or analyzed during this study are included in this published article.

\section{Authors' contributions}

FB performed the brain radiological examinations. LG, MM and VA performed the histological examinations of the lung tissue and contributed the histopathological details written in the manuscript. AR, AV and TM managed the cardiac complication of the patient and were involved in the collection and analysis of clinical data regarding the evolution of the cardiac disease. FP, AB, FF and MT analyzed patient's data and wrote the manuscript. All authors read and approved the final manuscript.

\section{Ethics approval and consent to participate}

Not applicable.

\section{Patient consent for publication}

Consent for publication was obtained verbally from patient's next of kin.

\section{Competing interests}

The authors declare that they have no competing interests.

\section{References}

1. Caine JG, Stonelake PS, Lip GYH and Kehoe ST: The hypercoagulable state of malignancy: Pathogenesis and current dabate. Neoplasia 4: 465-473, 2002.

2. Carrier M, Lazo-Langner A, Shivakumar S, Tagalakis V, Zarychanski R, Solymoss S, Routhier N, Douketis J, Danovitch K, Lee AY, et al: Screening for occult cancer in unprovoked venous thromboembolism. New Engl J Med 373: 697-704, 2015.

3. Abdol Razak NB, Jones G, Bhandari M, Berndt MC and Metharom P: Cancer-associated thrombosis: An overview of mechanisms, risk factors, and treatment. Cancers (Basel) 10: 380, 2018.

4. Aronson D and Brenner B: Arterial thrombosis and cancer. Thromb Res 164 (Suppl 1): S23-S28, 2018.

5. Lip GYH, Chin BSP and Blann AD: Cancer and the prothrombotic state. Lancet Oncol 3: 27-34, 2002.

6. el-Shami K, Griffiths E and Streiff M: Nonbacterial thrombotic endocarditis in cancer patients: Pathogenesis, diagnosis, and treatment. Oncologist 12: 518-523, 2007.

7. Mukai M and Oka T: Mechanism and management of cancer-associated thrombosis. J Cardiol 72: 89-93, 2018.

8. Horsted F, West J and Grainge MJ: Risk of venous thromboembolism in patients with cancer: A systematic review and meta-analysis. PLoS Med 9: e1001275, 2012.

9. Haddad TC and Greeno EW: Chemotherapy-induced thrombosis. Thrombo Res 118: 555-568, 2006.

10. Connolly G and Francis CW: Cancer-associated thrombosis. Hematology Am Soc Hematol Educ Program 2013: 684-691, 2013.

11. Raskob GE, van Es N, Verhamme P, Carrier M, Di Nisio M, Garcia D, Grosso MA, Kakkar AK, Kovacs MJ, Mercuri MF, et al: Edoxaban for treatment of cancer-associated venous thromboembolism. N Engl J Med 378: 615-624, 2018. 
12. Amin MB, Edge SB, Greene FL, Byrd DR, Brookland RK, Washington MK, Gershenwald JE, Compton CC, Hess KR, Sullivan DC, et al: AJCC Cancer Manual. 8th edition. Springer, New York, NY, 2017.

13. Coons AH, Creech HJ and Jones RN: Immunological properties of an antibody containing a fluorescent group. Exp Biol Med 47: 200-202, 1941.

14. Vallée A, Le Loupp A-G, Denis MG. Efficiency of the Therascreen ${ }^{\circledR}$ RGQ PCR kit for the detection of EGFR mutations in non-small cell lung carcinomas. Clin Chim Acta 429: $8-11,2014$

15. Reck M, Rodríguez-Abreu D, Robinson AG, Hui R, Csőszi T, Fülöp A, Gottfried M, Peled N, Tafreshi A, Cuffe S, et al: Pembrolizumab versus chemotherapy for PD-L1-positive non-small-cell lung cancer. N Engl J Med 375: 1823-1833, 2016.

16. Saenger AK, Beyrau R, Braun S, Cooray R, Dolci A, Freidank H, Giannitsis E, Gustafson S, Handy B, Katus H, et al: Multicenter analytical evaluation of a high-sensitivity troponin $\mathrm{T}$ assay. Clin Chim Acta 412: 748-754, 2011.

17. Mok Y, Ballew SH, Bash LD, Bhatt DL, Boden WE, Bonaca MP, Carrero JJ, Coresh J, D'Agostino RB Sr, Elley CR, et al: International validation of the thrombolysis in myocardial infarction (TIMI) risk score for secondary prevention in post-MI patients: A collaborative analysis of the chronic kidney disease prognosis consortium and the risk validation scientific committee. J Am Heart Assoc 7: e008426, 2018.

18. Layden J, Michaels J, Bermingham S and Higgins B; Guideline Development Group: Diagnosis and management of lower limb peripheral arterial disease: Summary of NICE guidance. BMJ 345: e4947, 2012.

19. Quintela AG, Candela MJ, Vidal C, Román J and Aramburo P: Non-bacterial thrombotic endocarditis in cancer patients. Acta Cardiol 46: 1-9, 1991.

20. Biller J, Challa VR, Toole JF and Howard VJ: Nonbacterial thrombotic endocarditis. A neurologic perspective of clinicopathologic correlations of 99 patients. Arch Neurol 39: 95-98, 1982.

21. Barry WE and Scarpelli D: Nonbacterial thrombotic endocarditis. A clinicopathologic study. Arch Intern Med 109: 79-84, 1962.

22. Liu J and Frishman WH: Nonbacterial thrombotic endocarditis: Pathogenesis, diagnosis and management. Cardiol Rev 24 244-247, 2016.

23. Kooiker JC, MacLean JM and Sumi SM: Cerebral embolism, marantic endocarditis, and cancer. Arch Neurol 33: 260-264, 1976.
24. Mazokopakis EE, Syros PK and Starakis IK: Nonbacterial thrombotic endocarditis (marantic endocarditis) in cancer patients. Cardiovasc Hematol Disord Drug Targets 10: 84-86, 2010.

25. Joffe II, Jacobs LE, Owen AN, Ioli A and Kotler MN: Noninfective valvular masses: Review of the literature with emphasis on imaging techniques and management. Am Heart J 131: 1175-1183, 1996.

26. Dutta T, Karas MG, Segal AZ and Kizer JR: Yield of transesophageal echocardiography for nonbacterial thrombotic endocarditis and other cardiac sources of embolism in cancer patients with cerebral ischemia. Am J Cardiol 97: 894-898, 2006.

27. Abouarab AA, Elmously A, Leonard JR, Arisha MJ, Gaudino M, Narula N and Salemi A: Nonbacterial trombotic endocarditis presenting with leg pain and a left atrial mass lesion. Cardiology 139: 208-211, 2018

28. Asopa S,Patel A, Khan OA, Sharma R and Ohri SK: Non-bacterial thrombotic endocarditis. Eur J Cardiothorac Surg 32: 696-701, 2007.

29. Lee AY, Levine MN, Baker RI, Bowden C, Kakkar AK, Prins M, Rickles FR, Julian JA, Haley S, Kovacs MJ, et al: Low-molecular-weight heparin versus a coumarin for the prevention of recurrent venous thromboembolism in patients with cancer. N Eng J Med 349: 146-153, 2003.

30. Franco-Moreno A, Cabezòn-Gutierréz L, Palka-Kotlowsa M, Villamayor-Delgado M and García-Navarro M: Evaluation of direct oral anticoagulants for the treatment of cancer-associated thrombosis: An update. J Thromb Thrombolysis 47: 409-419, 2019.

31. Fournier JB and Testa EJ: Nonbacterial thrombotic endocarditis. N Engl J Med 380: e48, 2019.

32. Lee RJ, Bartzokis T, Yeoh TK, Grogin HR, Choi D and Schnittger I: Enhanced detection of intracardiac source of cerebral emboli by transesophageal echocardiography. Stroke 22: 734-739, 1991.

33. Brosseau S, Gounant V, Naltet C, Théou-Anton N, Cazes A, Smonig R, Neuville M, Khalil A, Mikail N, Meignin V, et al: Lazarus syndrome with crizotinib in a non-small cell lung cancer patient with ROS1 rearrangement and disseminated intravascular coagulation. Clin Lung Cancer 19: e57-e61, 2018.

This work is licensed under a Creative Commons Attribution-NonCommercial-NoDerivatives 4.0 International (CC BY-NC-ND 4.0) License. 